\title{
CONSECUENCIAS Y USO DE LAS TIC ANTES Y DESPUÉS DEL CORONAVIRUS: UN ESTUDIO PILOTO
}

\author{
Patricia González Elices \\ Facultad de Ciencias de la Salud y Educación \\ Universidad a Distancia de Madrid \\ patricia.gonzalez@udima.es
}

\section{RESUMEN}

En un contexto en el que la llegada de la COVID-19 ha supuesto un reto educativo: la enseñanza a distancia, es necesario valorar si la decisión de adaptar la situación a un entorno online ha supuesto una oportunidad para dejar atrás una educación obsoleta y si la utilización de las TIC como medio para la enseñanza finalmente va a conseguir consolidarse, siendo importante conocer también si esta adaptación ha supuesto un factor estresante para los docentes que de un día para otro han tenido que modificar su forma de enseñar. Con el objetivo de conocer y dar respuesta a la pregunta señalada como título se realizó una pequeña investigación entre docentes en activo que hubiesen dado clase durante el confinamiento. La finalidad era poder contrastar el uso que hacían de las TIC antes, durante y después de esta situación y conocer el grado de estrés que supuso para ellos modificar su enseñanza. Para ello se elaboró un cuestionario que se difundió a través de la técnica de Bola de Nieve y se analizó con el SPSS. Los resultados indican que si bien algunos docentes mostraron niveles de estrés y nerviosismo al tener que modificar la forma de dar sus clases el concepto de las TIC y su utilización como medio para la enseñanza es positivo. La experiencia de una enseñanza virtual les ha motivado para seguir utilizando las TIC en sus clases presenciales y, consideran que la pandemia ha servido para consolidarlas y aumenta su uso en la docencia. Parece, por tanto, que las TIC finalmente han encontrado ese hueco prometido en el mundo educativo. Asimismo, si bien en la actualidad se promueve la formación digital entre los docentes, será necesario incidir más en este tipo de capacitación para que su inclusión no sea considerada como fuente de estrés.

Palabras clave: TIC; educación; enseñanza online; COVID-19; salud mental

\section{ABSTRACT}

Has the pandemic, and consequently the need to use ICT, been a positive or stressful factor for teaching?. In a context in which the arrival of COVID-19 has meant an educational challenge: distance 


\section{CONSECUENCIAS Y USO DE LAS TIC ANTES Y DESPUÉS DEL CORONAVIRUS: UN ESTUDIO PILOTO}

learning, it is necessary to assess whether the decision to adapt the situation to an online environment has been an opportunity to leave behind an obsolete education and whether the use of ICT as a means of teaching will finally consolidate, and it is also important to know whether this adaptation has been a stress factor for teachers who have had to change their way of teaching from one day to the next. In order to find out and give an answer to the question mentioned in the title, a small investigation was carried out among active teachers who had taught during the confinement. The aim was to compare their use of ICT before, during and after this situation and to find out the degree of stress they felt when modifying their teaching. To this end, a questionnaire was developed and disseminated using the Snowball technique and analyzed with SPSS. The results indicate that although some teachers showed levels of stress and nervousness at having to modify the way they teach their classes, the concept of ICT and its use as a medium for teaching is positive. The experience of virtual teaching has motivated them to continue using ICTs in their face-to-face classes, and they consider that the pandemic has served to consolidate them and increase their use in teaching. It seems, therefore, that ICTs have finally found their promised niche in the educational world. Likewise, although digital training is currently being promoted among teachers, it will be necessary to focus more on this type of training so that their inclusion is not considered a source of stress.

Keywords: information and communication technologies; education; online teaching; COVID-19; pandemic

\section{INTRODUCCIÓN}

En diciembre de 2019 se originó en China el Coronavirus SARS-CoV-2 que ha causado la pandemia de COVID-19 con efectos devastadores para la sociedad (Yi, Lagniton, Ye, Li y Xu, 2020) haciendo que el mundo, en su globalidad, se haya visto inmerso en incertidumbre y estrés. Todos los ámbitos se han visto afectados y entre ellos, la educación. Este sector ha visto como de un día para otro se cerraban los centros escolares paralizando la enseñanza y teniendo que buscar medidas alternativas. El cierre de las instituciones educativas Ilevó a que los docentes tuvieran que dejar sus clases, ese espacio tradicional al que estaban acostumbrados, para convertirse de manera obligada en usuarios de las herramientas tecnológicas que les permitiesen trasladar las clases del aula a las casas de sus estudiantes. Este cambio drástico debía además compaginarse con la propia presión personal que suponía para cada uno el propio confinamiento. Esta combinación simultánea sin duda ha creado un cóctel de emociones entre la comunidad de profesores pudiendo causar efectos múltiples en el proceso educativo.

Las clases remotas o teleformación han exigido que los docentes tuviesen que implementar cambios en sus estrategias de enseñanza y que se adaptasen a un contexto nuevo que ha dado lugar a diversos debates sobre la salud mental de los involucrados. La revisión realizada por Ribeiro, Scorsolini-Comin y Dalri, (2020) muestra los riesgos psicosociales que los docentes pueden padecer debido a la pandemia de COVID-19 y la consiguiente necesidad de realizar investigaciones para conocer los posibles efectos psicológicos que pueden derivarse del cambio que ha supuesto el desafío de modificar la enseñanza presencial a una enseñanza a distancia. Bajo esta línea otros estudios (Faro et al., 2020) han indicado un aumento de niveles de ansiedad, estrés o miedo en la población general.

Las medidas tomadas para mantener la formación en las distintas etapas educativas, suponen la necesidad de una competencia adaptativa docente muy significativa que puede suponer una sobrecarga emocional. Así mismo, la percepción de no estar suficientemente cualificado o la necesidad de compartir espacio con compañeros, cónyuges o hijos mientras se imparte la docencia pueden promover el sufrimiento emocional y verse afectada su salud mental (Ribeiro et al., 2020).

Estudios anteriores a la pandemia (Castillo, Fernández y López, 2014) ya revelaban la relación entre estrés, síntomas de ansiedad y cansancio emocional y la profesión docente. En esta línea, el estudio de Chakravorty (1989, citado en Franco, Mañas y Justo, 2016) reveló que un alto porcentaje (77\%) de las bajas laborales de larga duración se debían a patologías mentales relacionadas con el estrés docente. Así mismo, una investigación más reciente (Extremera, Durán y Rey, 2010) muestra que el síntoma mayoritario que padecen los profesores de Primaria y Secundaria es el cansancio emocional, encontrándose el 21,5\% en niveles severos. 
El bajo conocimiento teórico de los sotfware y hardware educativos implica una percepción negativa de las capacidades personales y una baja autoeficacia tecnológica que conduce al docente al incremento de estrés y ansiedad al considerarse no apto para atender las demandas de las Tecnologías de la Información y Comunicación, TIC (Gómez-López y Escoriaza, 2011) lo que puede influir en la utilización que hacen de estos recursos en sus aulas y a mantener un modelo de enseñanza tradicional. La tecnificación de la enseñanza supone la presencia de las TIC como modelo de enseñanza diferente, lo que supone para algunos docentes rechazo y temor al tener que utilizar estas tecnologías (tecnofobia) y repercute en un mayor nivel de estrés y consecuencias para la salud (Martínez-Otero, 2003).

La libertad de cátedra permite a los docentes el derecho a decidir cómo enseñar, siendo lógico pensar que una persona con tecnofobia utilizará en escasas ocasiones las TIC en sus clases. El problema deriva entonces cuando esa libertad se ve limitada viéndose obligados a incorporar las TIC en su docencia. Además, hay que sumar las propias dificultades técnicas que este tipo de recursos puede llevar aparejadas y que también pueden ser fuente de estrés, como, por ejemplo, la asincronicidad entre imagen y audio, la pérdida de conexión, ruidos distractores provenientes de la convivencia, etc., propios de la videoconferencia en tiempo real (García-García, 2020). Supone, por tanto, un reto la implantación de las TIC en la educación actual a la vez que se superan los obstáculos relacionados con el pensamiento docente: actitudes, creencias y expectativas (Gómez-López y Cano, 2011). En este sentido, la utilización de las TIC requiere la adquisición de competencias digitales, no obstante, Díaz-Arce y Loyola-Illescas (2021) refieren que a día de hoy los docentes no tienen aún las competencias digitales desarrolladas para hacer frente a esta digitalización.

En este contexto cabe preguntarse si este reto educativo y la decisión de adaptar la enseñanza a un entorno online ha supuesto una oportunidad para dejar atrás una educación obsoleta y si la utilización de las TIC, como medio para la enseñanza, finalmente va a conseguir consolidarse fomentando una mejora docente. Las medidas tomadas para mantener la formación en las distintas etapas educativas, suponen la necesidad de una competencia adaptativa docente muy significativa que puede suponer una sobrecarga emocional. Es fundamental, por tanto, también reflexionar sobre el proceso de adaptación que han realizado, explorando si esa adaptación ha supuesto un factor estresante para ellos. Si bien hay un exceso de información sobre los efectos de la pandemia hay muy poca información sobre los efectos que está teniendo en los docentes la realidad a la que se enfrentan para seguir ofreciendo una educación de calidad.

\section{OBJETIVOS DE LA INVESTIGACIÓN}

Con el objetivo de conocer los posibles efectos negativos, a nivel de estrés 0 ansiedad, que pudieron padecer los docentes durante la pandemia por tener que usar obligatoriamente las TIC se realizó una pequeña investigación a modo de piloto entre profesores en activo que hubiesen dado clase durante el confinamiento domiciliario. La finalidad era poder contrastar el uso que hacían de las TIC antes, durante y después de esta situación y conocer el grado de estrés que supuso para ellos modificar la modalidad de enseñanza. Esta investigación pretende, por ende, dar respuesta a cómo han vivido los docentes este cambio y qué ocurrirá después de la pandemia cuando se normalice nuevamente el sector educativo a la vez que pretende conocer si el uso de las TIC se incrementará en las aulas cotidianas consolidándose finalmente como recurso educativo.

\section{MÉTODO}

\section{Participantes}

La muestra de estudio quedó conformada por 60 docentes que quisieron formar parte del estudio de forma voluntaria y anónima. Las edades estuvieron comprendidas entre los 26 y los 59 años ( $M=44,24,=9,24), 33$ hombres (55\%) y 27 mujeres (45\%).

Los participantes procedían de 4 comunidades autónomas: 21 de la Comunidad de Madrid (35\%), 12 de Cataluña (20\%), 10 de Galicia (16,67\%), 9 de la Comunidad Valenciana (15\%) y 8 de Andalucía (13,33\%). 


\section{CONSECUENCIAS Y USO DE LAS TIC ANTES Y DESPUÉS DEL CORONAVIRUS: UN ESTUDIO PILOTO}

En relación con la etapa educativa 19 docentes (31,67\%) impartían clase en un nivel de enseñanza elemental (Infantil y Primaria), 22 (36,67) lo hacían a nivel medio (Secundaria y FP) y $19(31,67 \%)$ impartían docencia en la enseñanza de nivel avanzado (Universidad).

\section{Metodología}

Se utilizó un muestreo no probabilístico por conveniencia. Se recogieron inicialmente 69 respuestas, de las que se descartaron 9, 6 por no cumplir los requisitos (no haber estado ejerciendo la docencia durante el confinamiento) y 3 para tener una muestra cercana a los 20 participantes para cada etapa educativa que se quería analizar para poder hacer un análisis comparativo entre ellas en caso de que se viesen datos muy dispares en las variables de estudio.

Para poder administrar de forma autoadministrada el cuestionario se utilizó un formato online a través de Google Forms. La encuesta estuvo abierta 7 semanas, momento en que se alcanzó el porcentaje de participantes que se había establecido. La idea era hacer un estudio piloto para explorar posibles efectos de la utilización forzada de las TIC por el confinamiento para, en su caso, continuar posteriormente con la investigación depurando la encuesta centrándola en los aspectos significativos.

La solicitud de participantes se realizó a través de los contactos de la investigadora principal quien pidió a diferentes amigos docentes que cumplimentasen el formulario y que procurasen que se cumplimentara en su centro educativo. Tras la recogida de datos se depuró la hoja de cálculo exportada del formulario y se elaboró la base de datos correspondiente.

Los análisis estadísticos descriptivos e inferenciales se realizaron con el IBM SPSS 22.

\section{Instrumento}

Para la recogida de datos se empleó una encuesta elaborada ad hoc dividida en cinco secciones. La primera, a modo de cabecera, se explicaban las condiciones y objetivos del estudio y se garantizaba el anonimato y la protección de datos de los participantes. La segunda sección estaba confeccionada con preguntas de carácter demográfico (edad, sexo, Comunidad Autónoma en la que trabajaba, tipo de institución -pública, privada o concertada- y, etapa educativa. En esta sección además había dos preguntas que nos permitieron hacer de filtro para saber si los docentes ejercían la docencia antes de la pandemia y si estaban o no activos durante el confinamiento. Para poder seguir el cuestionario los participantes debían necesariamente marcar en las dos cuestiones "si", de lo contrario el formulario avanzaba hasta a la última página en la cual se agradecía su participación y se explicaba el motivo de no pertenecer a la muestra de estudio. La tercera sección se dividía a su vez en dos bloques. En el primero se preguntaba por el uso que hacían de las TIC antes de la pandemia mientras que en el segundo se solicitaba que marcasen el uso que hacían después del confinamiento. En la cuarta sección se presentaban también dos bloques de preguntas para ser respondidas en formato likert señalando su grado de acuerdo o desacuerdo con las distintas afirmaciones propuestas. Las opciones tomaban valores desde 1 "nada de acuerdo" a 5 "completamente de acuerdo". Finalmente, la quinta y última sección pretendía recoger datos sobre un cambio de concepción en relación con las TIC y sobre formación docente con finalidad de una mejora en competencia digital.

\section{RESULTADOS}

La Figura 1 muestra una comparativa en relación al uso que los docentes participantes realizaban antes de la pandemia y después del confinamiento. Como puede observarse hay un cambio en relación con la frecuencia e intención de uso, así, un 31,67\% indicó que antes del confinamiento utilizaba las TIC todos los días incrementándose hasta el $50 \%$ cuando se les pregunta por el momento actual y la intención en cuanto vuelvan al centro educativo. Un 33,3\% marcaron que las utilizaban asiduamente (varios días a la semana) antes del confinamiento siendo el 20\% quien reflejó esta intención tras la vuelta a las aulas. La tendencia es similar con la respuesta una vez a la semana, un $10 \%$ las utilizan antes y un $8,33 \%$ indican hacerlo después. A frecuencia una 0 dos veces al mes no varía ( $5 \%$ en ambos casos), mientras que la respuesta "apenas usaba las TIC" es contestado por el 8,33\% 
antes del COVID reduciéndose al 1,67\% después. Por último, únicamente un participante indicó no usar las TIC antes de la pandemia $(1,67 \%)$ quedando sin ser elegida esta opción cuando se les pregunta por la intención al regreso de la normalidad escolar.

Figura 1. Uso de las TIC antes y después de la pandemia.

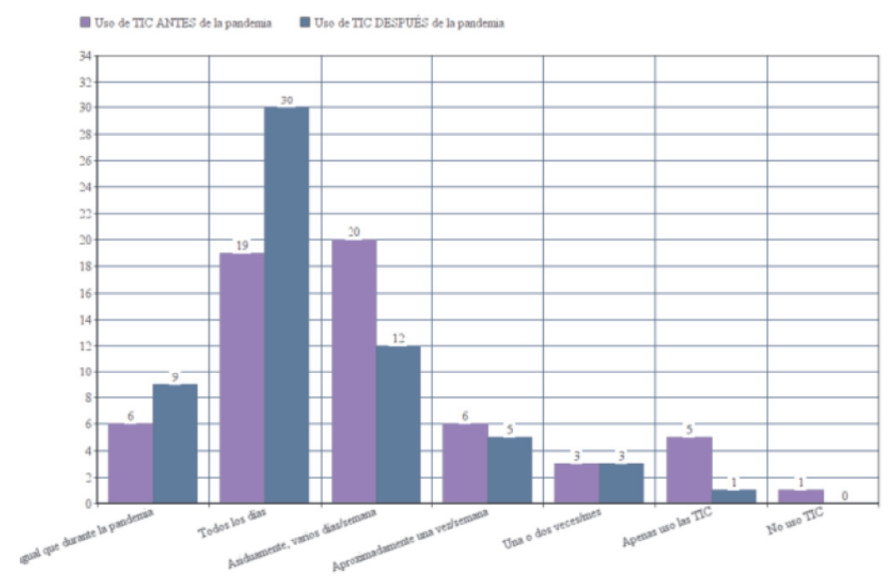

En relación con la valoración del uso de las TIC para la docencia (Tabla 1) se observa que prácticamente la mitad de la muestra (46,6\%) no consideran que el haber tenido que utilizar la enseñanza online haya supuesto una oportunidad para dejar atrás una educación obsoleta, aunque el 50\% indican que consideran que las TIC han cambiado positivamente la docencia. Por otro lado, la opinión mayoritaria de los participantes considera que las TIC han Ilegado para quedarse y que no se puede volver al modelo tradicional (60\%) a la vez que están de acuerdo con que el uso de estas herramientas va a suponer su consolidación (65\%).

En este orden de ideas, 28 participantes (46\%) han indicado que no ha cambiado su concepción de las TIC con respecto a antes de la pandemia mientras que un 53\% (32 participantes) indicaron que sí.

Tabla 1. Valoración del uso de las TIC para la docencia

\begin{tabular}{|c|c|c|}
\hline Afirmación & $\begin{array}{c}\text { Escala de } \\
\text { valor }\end{array}$ & Valor (\% ) \\
\hline \multirow{2}{*}{$\begin{array}{l}\text { Considero que el haber tenido que utilizar } \\
\text { un entorno online ha supuesto una } \\
\text { oportunidad para dejar atrás una } \\
\text { educación obsoleta }\end{array}$} & Nada o poco & $28(46,67 \%)$ \\
\hline & $\begin{array}{l}\text { Mucho o } \\
\text { totalmente }\end{array}$ & $14(23,33 \%)$ \\
\hline \multirow{2}{*}{$\begin{array}{l}\text { Considero que las TIC han llegado para } \\
\text { quedarse (ahora ya no se puede volver al } \\
\text { modelo tradicional) }\end{array}$} & Nada o poco & $12(20 \%)$ \\
\hline & $\begin{array}{l}\text { Mucho o } \\
\text { totalmente }\end{array}$ & $36(60 \%)$ \\
\hline \multirow{2}{*}{$\begin{array}{l}\text { La utilización de las TIC como como } \\
\text { medio para la enseñanza finalmente va a } \\
\text { conseguir consolidarse }\end{array}$} & Nada o poco & $7(11,67 \%)$ \\
\hline & $\begin{array}{l}\text { Mucho o } \\
\text { totalmente }\end{array}$ & $39(65 \%)$ \\
\hline \multirow{2}{*}{$\begin{array}{l}\text { Las TIC han cambiado positivamente la } \\
\text { docencia }\end{array}$} & Nada o poco & $11(18,33 \%)$ \\
\hline & $\begin{array}{l}\text { Mucho o } \\
\text { totalmente }\end{array}$ & $30(50 \%)$ \\
\hline
\end{tabular}




\section{CONSECUENCIAS Y USO DE LAS TIC ANTES Y DESPUÉS DEL CORONAVIRUS: UN ESTUDIO PILOTO}

Por su parte, la Tabla 2 ofrece un resumen de los datos obtenidos en relación con el uso de las TIC como factor estresante durante la pandemia. Como puede observarse un 35\% de los docentes encuestados indicaron sentir estrés cuando se enteraron que tenían que realizar clases online. El mismo porcentaje indica igualmente que se han sentido perdidos por el desconocimiento de algunas herramientas tecnológicas, mientras que el $30 \%$ comunica sentirse inquieto por su nivel de competencia digital y el 20\% sentirse nervioso por no saber cómo controlar la clase. Finalmente el 21,67\% de la muestra indicó estar más relajado si no tenía que utilizar herramientas tecnológicas para dar clase.

Tabla 2. Uso de las TIC durante la pandemia como factor estresante

\begin{tabular}{|c|c|c|}
\hline Afirmación & $\begin{array}{l}\text { Escala de } \\
\text { valor }\end{array}$ & Valor (\%) \\
\hline \multirow{2}{*}{$\begin{array}{l}\text { Senti gran estrés cuando me comunicaron } \\
\text { que tenía que dar las clases online }\end{array}$} & Nada o poco & $26(43,33 \%)$ \\
\hline & $\begin{array}{l}\text { Mucho o } \\
\text { totalmente }\end{array}$ & $21(35 \% \%)$ \\
\hline \multirow{2}{*}{$\begin{array}{l}\text { Me he sentido perdido/perdida por el } \\
\text { desconocimiento de algunas herramientas } \\
\text { tecnológicas }\end{array}$} & Nada o poco & $34(56,67 \%)$ \\
\hline & $\begin{array}{l}\text { Mucho o } \\
\text { totalmente }\end{array}$ & $21(35 \%)$ \\
\hline \multirow{2}{*}{$\begin{array}{l}\text { Me sentia inquieto/a por mi nivel de } \\
\text { competencia digital (y las posibles } \\
\text { diferencias con mis estudiantes) }\end{array}$} & Nada o poco & $38(63,33 \%)$ \\
\hline & $\begin{array}{l}\text { Mucho o } \\
\text { totalmente }\end{array}$ & $20(30 \%)$ \\
\hline \multirow{2}{*}{$\begin{array}{l}\text { Me sentia nervioso/a por no saber cómo } \\
\text { controlar la clase }\end{array}$} & Nada o poco & $33(55 \%)$ \\
\hline & $\begin{array}{l}\text { Mucho o } \\
\text { totalmente }\end{array}$ & $12(20 \%)$ \\
\hline \multirow{2}{*}{$\begin{array}{l}\text { Me siento más relajadalo al no tener que } \\
\text { utilizar herramientas tecnológicas para } \\
\text { dar clase }\end{array}$} & Nada o poco & $35(58.33 \%)$ \\
\hline & $\begin{array}{l}\text { Mucho o } \\
\text { totalmente }\end{array}$ & $13(21,67 \%)$ \\
\hline
\end{tabular}

En relación con la competencia digital además señalar que el $65 \%$ de los participantes indicó que durante la pandemia se han apuntado a cursos o seminarios para mejorar su nivel.

\section{DISCUSIÓN Y CONCLUSIONES}

Son múltiples los cambios a los que debe someterse la sociedad y más, en la situación pandémica actual. La educación debe adaptarse a estas transformaciones de la forma más positiva posible minimizando las posibles consecuencias. Como se vio en la introducción, hacer frente a los cambios, utilización y, actualización y aprendizaje de las TIC, puede suponer un esfuerzo constante que repercute en la salud mental. La capacidad de adaptación docente es la clave para controlar el nivel de estrés que provocan estas situaciones (Ayuso, 2006) y si bien la organización del tiempo, entre el trabajo, el descanso y otras actividades diarias, se ha marcado como un factor de prevención (Ribeiro et al., 2020) durante el confinamiento se ha visto dificultada pudiendo incrementar el nivel de estrés entre los profesores. Los hallazgos de la presente investigación, sin embargo, muestran que los docentes españoles se han adaptado bastante bien a la enseñanza online. Los resultados muestran que si bien algunos docentes mostraron niveles de estrés y nerviosismo al tener que modificar la forma de dar sus clases el concepto de las TIC y su utilización como medio para la docencia es positivo. En esta línea, y de forma similar a los resultados descritos en el estudio de Díaz-Arce y Loyola-IIlescas (2021), parece que los docentes no perciben como un factor excesivamente estresante el manejo de herramientas tecnológicas o el control de los grupos a distancia, siendo, por contra, un colectivo propenso al sufrimiento emocional (Ribeiro, et al., 2020). 
Compartimos con Gisbert (2000, citado en Gómez-López, 2011) que el principal factor estresor puede provenir de modificar el rol tradicional de enseñanza y de la denominada resistencia al cambio, no obstante, los resultados de esta investigación señalan que la experiencia de una enseñanza virtual les ha motivado para seguir utilizando las TIC en sus clases presenciales a la vez que consideran que la pandemia ha servido para consolidarlas y aumentar su uso en docencia. Así mismo, si bien en la actualidad se promueve la formación digital entre los docentes y parece que la percepción mayoritaria entre eta población es que su nivel de competencia es suficiente (o por lo menos tal para no causar demasiada inquietud) parece necesario incidir más en este tipo de capacitación para que su inclusión no sea considerada como factor negativo para la salud mental. Esta conclusión parece estar en consonancia también con los resultados de Gómez-García, Hinojo-Lucena, Cáceres-Reche y Ramos (2020) quienes revelan que el tema de las competencias digitales docentes es prioritario para poder implementar una metodología de enseñanza diferente.

La educación presencial ya no será la misma después de la pandemia ya que muchos docentes optarán por continuar sus clases haciendo uso de las TIC. Los datos muestran que a pesar de que los docentes antes de la pandemia ya hacían bastante uso de las TIC, (el 65\% informaba de usar varias veces por semana 0 incluso todos los días estas tecnologías) el uso forzoso del confinamiento para poder realizar la docencia online ha provocado que esta tendencia se incremente hasta el 70\%, si bien es cierto que todo parece indicar que los cambios se personalizan en aquellos profesores que ya las utilizaban, se aprecia también un cambio favorable de los docentes que señalaban hacer un uso nulo o infrecuente de las TIC antes del COVID-19. En este sentido el porcentaje decrece significativamente (del 10\% al 1,67\%). En esta línea los hallazgos parecen señalar que los mayores cambios se realizarán en la etapa de enseñanza de nivel medio (pues han sido los profesores de Secundaria o FP quienes mostraron mayores diferencias). Estos datos, junto con los resultados relacionados con la actitud, formación y consideración global frente a las TIC parecen estar en consecuencia con la necesidad de adaptar la práctica docente, fortaleciendo los procesos de enseñanza-aprendizaje y actualizando sus conocimientos sobre aspectos tecnológicos, en correspondencia con los resultados de Sandoval (2020). El hecho de que el 65\% de los docentes muestre interés por mejorar su competencia digital respalda los hallazgos de (Tejedor, Cervi, Tusa, y Parola, 2020) los cuales mostraban que durante la teleformación los profesores deben tener conocimientos tecnológicos y digitales. Se cuenta así con una apertura docente hacia nuevos recursos, metodologías y una actitud positiva para actualizar la formación presencial incrementando el uso de las TIC. Sin embargo, no debemos olvidar que esta implementación supone un esfuerzo adicional de los docentes, responsables de aplicarlas de forma correcta y pedagógica en el entorno educativo compatibilizando sus tareas lectivas con esta formación en competencias digitales, teniendo que asumir esta carga extra de trabajo. Si bien los resultados de este pequeño estudio no ofrece datos preocupantes sobre el nivel de estrés percibido es fundamental que se tengan en cuenta estos factores que, sumados a la falta de información y de tiempo, y a la tecnofobia o miedo a los cambios tecnológicos pueden suponer en un futuro un aumento de ansiedad y un detrimento de la salud mental de esta población. Por ello, se debe tener en cuenta, al igual que en cualquier proceso de implantación, los factores que pueden ser vividos como una sobrecarga emocional para planificar estrategias de escape y formación.

Por todo ello, compartimos la conclusión a la que llega Laro (2020): parece que finalmente las TIC han Ilegado para quedarse y que supondrán irremediablemente un cambio en el modelo actual. Motivo por el cual, y a la vista de los resultados de este pequeño estudio, parece necesario ampliar la investigación sobre los posibles efectos negativos que puede presentar una incorporación obligatoria y poco paulatina de las TIC para salvaguardar la salud mental de nuestros docentes, agentes prioritarios en la educación de la futura sociedad. En vista de lo expuesto, se advierte el imperativo de repensar en la salud de los profesionales de la educación no solo a nivel físico sino a nivel mental y promover estrategias de apoyo psicológico, ya que si su exposición al estrés laboral ya es significativa por los distintos factores psicosociales (Alvites-Huamaní, 2019) el actual escenario puede ser otro detonante importante. En esta línea para futuras investigaciones se recomienda contar con una muestra mayor que permita la generalización de resultados. 


\section{CONSECUENCIAS Y USO DE LAS TIC ANTES Y DESPUÉS DEL CORONAVIRUS: UN ESTUDIO PILOTO}

\section{REFERENCIAS BIBLIOGRÁFICAS}

Alvites-Huamaní, C. (2019). Estrés docente y factores psicosociales en docentes de Latinoamérica, Norteamérica y Europa. Propósitos y Representaciones, 7(3), 141-159. https://dx.doi.org/10.20511/pyr2019.v7n3.393

Ayuso, J. A. (2006). Profesión docente y estrés laboral: una aproximación a los conceptos de estrés laboral y burnout. Revista Iberoamericana de Educación, 1-14. http://www. rieoei. org/deloslectores/1341Ayuso. pdf.

Castillo, A., Fernández, R., \& López, P. (2014). Prevalencia de ansiedad y depresión en docentes. Revista Enfermería del Trabajo, 4(2), 55-62.

Extremera, N., Durán, A., \& Rey, L. (2010). Recursos personales, síndrome de estar quemado por el trabajo y sintomatología asociada al estrés en docentes de enseñanza primaria y secundaria. Ansiedad y estrés, 16(1), 47-60.

Chirinos, M. P., Olivera, N. A. G., \& Cerra, D. C. (2020). En tiempos de coronavirus: las TIC S son una buena alternativa para la educación remota. Revista Boletín Redipe, 9(8), 158-165.

Díaz-Arce, D., \& Loyola-IIlescas, E. (2021). Competencias digitales en el contexto COVID 19: una mirada desde la educación. Revista Innova Educación, 3(1), 120-150. https://doi.org/10.35622/j.rie.2021.01.006

Extremera, N., Durán, A., \& Rey, L. (2010). Recursos personales, síndrome de estar quemado por el trabajo y sintomatología asociada al estrés en docentes de enseñanza primaria y secundaria. Ansiedad y estrés, 16(1), 47-60.

Faro, A., Bahiano, M. D. A., Nakano, T. D. C., Reis, C., Silva, B. F. P. D., \& Vitti, L. S. (2020). COVID-19 e saúde mental: a emergência do cuidado. Estudos de Psicologia (Campinas), 37, e200074. Epub June 01, 2020.https://doi.org/10.1590/1982-0275202037e200074

Franco, C., Mañas, I., \& Justo, E. (2016). Reducción de los niveles de estrés, ansiedad y depresión en docentes de educación especial a través de un programa de mindfulness. Revista de Educación Inclusiva, 2(3), 11-33.

García-García, M. D. (2020). La docencia desde el hogar. Una alternativa necesaria en tiempos del Covid 19. Polo del Conocimiento: Revista científico-profesional, 5(4), 304-324. D0I: 10.23857/pc.v5i3.1318

Gómez-García, G., Hinojo-Lucena, F. J., Cáceres-Reche, M. P., \& Ramos Navas-Parejo, M. (2020). The Contribution of the Flipped Classroom Method to the Development of Information Literacy: A Systematic Review. Sustainability, 12(18), 7273. 1-13. D0l:10.3390/su12187273

Gómez-López, J., \& Cano, J. (2011). El pensamiento docente y su influencia en la implantación de las tecnologías de la información y la comunicación en el aula: desafíos y oportunidades. Contextos educativos. Revista de educación, (14), 67-84.

Laro, E. (2020). Innovar enseñando: la educación del futuro. Las TICs como factor motivador en la enseñanza. Revista Jurídica de Investigación e Innovación Educativa (REJIE Nueva Época), (21), 11-23. https://doi.org/10.24310/REJIE.2020.v0i21.7530

Martínez-Otero, V. (2003). Estrés y ansiedad en los docentes. Pulso: revista de educación, (26), 9-22.

Mendiola, M. S., Hernández, A., Torres, R., Carrasco, M., Romo, A., Mario, A., \& Cazales, V. (2020). Retos educativos durante la pandemia de COVID-19: una encuesta a profesores de la UNAM. Revista Digital Universitaria, 21(3), 1-24.

Moore, M. G. (2013). Teoría de la distancia transaccional. En D. Keegan (Ed.), Principios teóricos de la educación a distancia. Nueva York: Routledge.

Oña-Simbaña, J. M. (2020). Desafíos de la educación preescolar en tiempos de COVID-19. CienciAmérica, 9(2), 138-145.

Ramos-Huenteo, V., García-Vásquez, H., Olea-González, C., Lobos-Peña, K., \& Sáez-Delgado, F. (2020). Percepción docente respecto al trabajo pedagógico durante la COVID-19. CienciAmérica, 9(2), 334-353.

Reynosa Navarro, E., Rivera Arellano, E. G., Rodríguez Galán, D. B., \& Bravo Díaz, R. E. (2020). Adaptación docente educativa en el contexto covid-19: una revisión sistemática. Conrado, 16(77), 141-149.

Ribeiro, M., Scorsolini-Comin, F., \& Dalri, R. (2020). Ser docente en el contexto de la pandemia de COVID-19: 
reflexiones sobre la salud mental. Index de Enfermería, 29(3), 137-141. Epub 25 de enero de 2021. Recuperado en 3 de mayo de 2021, de http://scielo.isciii.es/scielo.php?script=sci_arttext\&pid=S1132$12962020000200008 \& \operatorname{lng}=e s \& t \operatorname{lng}=e s$.

Sandoval, C. (2020). La educación en tiempo del Covid-19 herramientas TIC: El nuevo rol Docente en el fortalecimiento del proceso enseñanza aprendizaje de las prácticas educativa innovadoras. Revista TecnológicaEducativa Docentes 2.0, 9(2), 24-31. https://doi.org/10.37843/rted.v9i2.138

Tejedor, S., Cervi, L., Tusa, F. y Parola, A. (2020). Educación en tiempos de pandemia: reflexiones de alumnos y profesores sobre la enseñanza virtual universitaria en España, Italia y Ecuador. Revista Latina, (78), 1-21. https://doi.org/10.4185/RLCS-2020-1466

Yi, Y., Lagniton, P. N., Ye, S., Li, E., \& Xu, R. H. (2020). COVID-19: what has been learned and to be learned about the novel coronavirus disease. International journal of biological sciences, 16(10), 1753-1766. https://doi.org/10.7150/ijbs.45134. 
